\title{
Evaluation of herd-level sampling strategies for control of Salmonella in Swedish cattle
}

\author{
E. C. C. Ågren, ${ }^{* 1}$ S. Sternberg Lewerin, $\dagger$ and J. Frössling* \\ ${ }^{*}$ Department of Disease Control and Epidemiology, National Veterinary Institute (SVA), SE-751 89 Uppsala, Sweden \\ †Department of Biomedical Sciences and Veterinary Public Health, SLU, Swedish University of Agricultural Sciences, Box 7036, \\ SE-750 07 Uppsala, Sweden
}

\section{ABSTRACT}

Based on Swedish legislation, all herds where Salmonella of any serotype is detected are put under restrictions, and measures aiming at eradication are required. Costs for sampling and control have increased in recent years and the aim of this study was to investigate the efficiency of different sampling strategies. We also compiled test results from recent surveillance activities and used these to complement and compare with calculated results. Sensitivities and specificities at group and herd level were calculated for different test strategies. A scenario-tree modeling approach was used to account for the hierarchy of animals within herds, and different relative risk of salmonella in different age groups. Negative and positive predictive values (NPV and PPV), and probability of freedom from Salmonella were calculated to compare the added value of different sampling strategies. Results showed that more fecal samples than serological samples per group are needed to reach a group sensitivity $>0.50$. This also means that serological testing leads to a higher NPV. For example, with 10 negative test-results from a group of 25 animals in a herd with a suspicion of Salmonella, the NPV based on serology was 0.75 and based on culture was 0.56 . For the PPV, testing based on culture from fecal sampling was superior, as specificity of such testing was close to perfect. By changing the threshold for considering a group positive, from 1 test-positive animal to 2 , the PPV of serological results could be increased without substantial loss in NPV. The herd sensitivity based on (1) bulk milk sampling, (2) fecal sampling of all animals, and (3) bulk milk sampling and individual sera from 20 animals within each age group was $0.53,0.88$, and 0.95 , respectively. In low-prevalence regions, this sensitivity was enough to verify a high probability of freedom $(>0.99)$, as the probability of infection in such Swedish regions has been shown to be 0.01 . For herds

Received March 21, 2018.

Accepted June 30, 2018.

${ }^{1}$ Corresponding author: estelle.agren@sva.se with a higher prior probability of infection, repeated sampling (2-9 sampling occasions) was needed to reach the same level of confidence. Analysis of surveillance data indicated that boot swabs can be used to replace the standard fecal sampling presently used in Sweden. It was also confirmed that the individual specificity of the tests used for serological testing of Swedish calves is high (0.99). The results can form a basis for fit-forpurpose testing strategies (e.g., surveillance or prepurchase testing).

Key words: animal health surveillance, freedom from infection, testing, bovine, Salmonella

\section{INTRODUCTION}

Salmonella is an important food-borne pathogen, and during the last decade increasing efforts have been made to control Salmonella in European animal production (Hugas and Beloeil, 2014). Although cattle are not the main source of infection, dairy products and beef are known sources for human salmonellosis (Pires et al., 2014). Some serotypes of Salmonella can cause clinical disease in cattle herds, in particular the host-adapted Salmonella Dublin that can cause serious disease and production losses (Richardson, 1975; Carrique-Mas et al., 2010; Nielsen et al., 2013). Control programs targeting Salmonella Dublin infection in cattle are present in some countries (Nielsen, 2012). In Sweden, a national control program for all Salmonella serotypes has been in place since the 1960s (National Veterinary Institute, 2017). In a recent Swedish bulk milk screening 3\% of the dairy herds were seropositive, a third of these were positive as regards to Salmonella Dublin (Ågren et al., 2016). These herds were geographically clustered in Öland, an island off the southeast coast of Sweden, where the proportion of test-positive herds was $15 \%$.

Assessment of infection status and classification of herds is an important part of control programs; it may form the basis for trading standards and also affect other restrictions and recommendations. The standard method to classify herds within the Swedish Salmonella control program is culture of fecal samples (Swedish 
Board of Agriculture, 2004). More recently, serological testing of bulk milk has been used for screening purposes and trace-back investigations. Individual serology has been used in the mandatory on-farm control in infected herds as part of monitoring the effect of implemented hygiene measures. In addition, serology is used within a new program for safe livestock trade, run by the Swedish dairy association (Växa Sverige, Sweden). Hence, individual animals and herds are frequently tested for Salmonella; however, the imperfect performance of available diagnostic tests can complicate the interpretation of results. Repeated sampling and different combinations of samples and tests are often used in attempts to improve the confidence in a herd's Salmonella status. The benefits of such testing have not been fully explored but can be expected to depend on several factors, namely the tests used, the number of animals sampled, age of the animals sampled, type of sample, prevalence, and the purpose of testing.

The aim of our study was to investigate the efficiency of different sampling strategies, at group level and at herd level, by performing calculations on different combinations of tests, sample sizes, and sampling frequencies, considering the most common scenarios in Swedish cattle herds. As part of this, we also compiled results from recent surveillance activities and used these to complement and compare with calculated results.

\section{MATERIALS AND METHODS}

\section{Calculations to Estimate Efficiency of Sampling Strategies}

The performance of different sampling strategies was estimated by calculating surveillance sensitivities and predictive values at group level, as well as sensitivities and probability of freedom from infection at herd level. A 3-level population structure was thus used, with animals within groups and groups within herds, and estimates were calculated for the aggregate levels (group or herd). In the calculations at herd level, we used the principle of scenario-tree modeling, described by Martin et al. (2007a,b) for demonstrating disease freedom in a population. All herds were assumed to consist of 3 groups of animals - calves, young stock, and adult animals - and estimates were calculated taking this herd structure into consideration. We use the term "infected" for the (unknown) true infection status of an individual, group or herd. The term "serology" was used for antibody testing of serum and of bulk milk. We decided input values for our calculations through consensus after considering relevant published data (Table 1). For sensitivities and specificities on animal level, estimates for culture were made in relation to current bacterial infection and for serology in relation to presence of antibodies. A range was used for these values and calculations were performed with 10,000 stochastic iterations using values from a $\beta$-PERT distribution. Calculations were performed in $\mathrm{R}$ ( $\mathrm{R}$ Development Core Team, 2018) using the package RSurveillance (Sergeant, 2016).

First, we calculated the sensitivity at group level (GrSe), which corresponds to the probability of detecting infection in a group, if present, at or above a specified design prevalence. The design prevalence $\left(\boldsymbol{P}^{*}\right)$ is the set threshold, or minimum proportion of infected animals. A group was considered test positive if at least 1 test-positive animal was present; 2 testpositive animals were used as an alternative threshold for serology. An approximation of the hypergeometric

Table 1. Input values in calculations used to compare efficiency of different sampling strategies for investigations of presence of Salmonella in cattle (values were determined by the authors after considering relevant references)

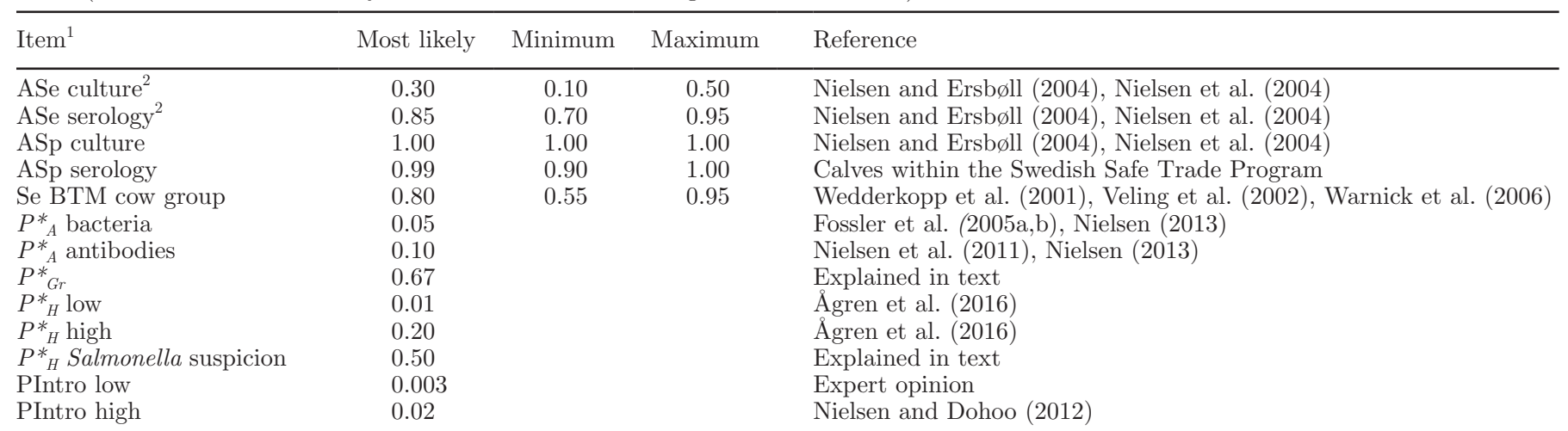

${ }^{1} \mathrm{ASe}=$ sensitivity at individual animal level; ASp = specificity at individual animal level; Se BTM cow group = sensitivity of a bulk milk sample to detect infection among the lactating cows; $P^{*}{ }_{A}=$ prevalence of infected animals in a group; $P^{*}{ }_{G r}=$ prevalence of infected groups in a herd; $P_{H}^{*}=$ prevalence of infected herds in a region; PIntro = probability of introduction of infection into a herd during a 3-mo period.

${ }^{2}$ The input for ASe of fecal culture and serology was set to higher values than in the references to adjust for difference in case definition. 
probability distribution was used for calculation of GrSe:

$$
G r S e=1-\left(1-\frac{A S e \times n}{N}\right)^{d},
$$

where $A S e$ is the diagnostic test sensitivity on the animal level, $n$ is the number of sampled animals, $N$ is the number of animals in the group, and $d$ is the assumed number of infected animals in the group. The number of infected animals in the group is $P^{*} \times N$ rounded off to the next integer, where $P^{*}$ is the within-group design prevalence.

The animal-level specificity was assumed to be 1 for all calculations of freedom from infection (PFree) at herd level regardless of type of diagnostic test. This is an accepted assumption in calculations of disease freedom (Martin et al., 2007a) that simplifies calculations and is a conservative approach that will help avoid overestimation of the probability of freedom. For calculations of sensitivity and predictive values at group level, the animal-level specificity was assumed to be perfect for testing based on isolation of Salmonella (i.e., from fecal culture). For calculations of GrSe based on testing with serology, an animal-level specificity less than 1 was used and GrSe was estimated from a more complex calculation using a modified hypergeometric probability distribution (Cameron and Baldock, 1998), as applied in the RSurveillance package (Sergeant, 2016).

For estimations at group level, we calculated the positive predictive value (PPV; the probability that a test-positive group is infected) and the negative predictive value (NPV; the probability that a test-negative group is not infected). The following calculations were used (Dohoo et al., 2009):

$$
\begin{gathered}
P P V=\frac{p(D+) \times G r S e}{p(D+) \times G r S e+p(D-) \times(1-G r S p)}, \text { and } \\
N P V=\frac{p(D-) \times G r S p}{p(D-) \times G r S p+p(D+) \times(1-G r S e)},
\end{gathered}
$$

where $p(D+)$ is the probability that a group is infected; $p(D-)$ is the probability that a group is not infected; and GrSp is group specificity. In the absence of positive test results and an assumed animal-level specificity of 1 , the NPV is equivalent to the postprobability of freedom from infection (PostPFree) at the level of the design prevalence.
The risk of being infected with Salmonella can be expected to vary between age categories. This was incorporated into the calculations at herd level by adjusting the within-group prevalence $\left(P^{*}\right)$ and calculating the effective probability of infection within each age category. In more detail, the relative risk of infection $\left(R R_{H R}\right)$ comparing a high-risk category, in this case calves and young stock, to a low-risk category, in this case cows, was included as adjusted risks in low risk groups $\left(A R_{L R}\right)$ and high risk groups $\left(A R_{H R}\right)$, taking into account both the relative risk and the proportion of animals within the different categories in the herd (PrPopLR and PrPopHR):

$$
\begin{gathered}
A R_{L R}=\frac{1}{R R_{H R} \times \operatorname{PrPopHR}+(1-\operatorname{PrPopHR})} \text {, and } \\
A R_{H R}=R R_{H R} \times A R_{L R} .
\end{gathered}
$$

For each stratum $(k)$ formed by the risk categories $(j$ $=1, \ldots, J)$, the design prevalence and the product of adjusted risks on animal level were used to calculate the effective probability of an animal being infected $\left(E P I n f_{k}\right)$ :

$$
E P \operatorname{Inf}_{k}=P_{A}^{*} \times \prod_{j=1}^{J} A R_{j} .
$$

This means that, although the total probability of infection (i.e., design prevalence) was kept constant, the effective probability of infection increased for animals in high-risk categories and decreased for animals in low-risk categories. This way, more weight is given to samples taken from high-risk individuals.

The difference in risk was estimated from published studies to be approximately twice as high in calves and young stock as in cows (2:2:1) (Fossler et al., 2005a,b; Nielsen, 2013). The proportion of animals within each age category was set to twice as many cows as calves and young stock respectively (1:1:2) according to statistics for Swedish dairy herds (Swedish Board of Agriculture, 2012).

In the interpretation at herd level of aggregated test results from individual animals, herd sensitivity (HSe) is the probability of detecting infection in a herd, if present, at or above the design prevalence. It was calculated by first combining all samplings $(l=1, \ldots, L)$ within the same group $(m=1, \ldots, M)$ by

$$
\mathrm{GrSeComb}_{m}=1-\prod_{l=1}^{L}\left(1-G r S e_{l}\right) \text {, }
$$


where $\mathrm{GrSeComb}$ is the combined group sensitivity. Then HSe was calculated by

$$
H S e=1-\prod_{m=1}^{M}\left[1-\left(P_{G r}^{*} \times G r S e \operatorname{Comb}_{m}\right)\right] .
$$

For strategies with only 1 type of sample per group, the $G r S e C o m b=$ GrSe. The design prevalence at group level $\left(P_{G r}^{*}\right)$ was set to $2 / 3$ to correspond to 2 infected age groups out of 3 groups in total. As an alternative, $P^{*}{ }_{G r}=1 / 3$ (i.e., 1 infected age group out of 3 groups) was also used. The PostPFree thus reflects how probable it is that the herd does not have Salmonellainfected animals at the within-group design prevalence, even if not present in all age groups. The probability of freedom from infection after testing for Salmonella (PostPFree), given that all test results are negative, was calculated as

$$
\text { PostPFree }=\frac{1-\text { PriorPInf }}{1-\text { PriorPInf } \times H S e},
$$

where PriorPInf is the herd's prior probability of infection. Three levels of PriorPInf were used: 0.01, 0.20 , and 0.50 . The 2 first levels equal the estimated prevalence of Salmonella Dublin in different regions in Sweden (Ågren et al., 2016). A prior of 0.50 was included to reflect the scenario of testing a herd with a nonconfirmed suspicion of infection (e.g., in trace-back investigations).

The added value of repeated sampling was calculated by temporal discounting of the probability of freedom from infection, where the prior probability of infection for the most recent time-period $t\left(\right.$ PriorPInf $\left._{\mathrm{t}}\right)$ was set to the posterior probability of infection (PostPInf $=$ 1 - PostPFree) achieved at the preceding time-period $(t-1)$. However, to adjust for the probability of introduction of Salmonella into the herd during one timeperiod (PIntro), this PriorPInf was upgraded using the equation

$$
\begin{gathered}
\text { PriorPInf }=\text { PIntro }+ \text { PostPInf }_{t-1} \\
- \text { PIntro } \times \text { PostPInf } f_{t-1},
\end{gathered}
$$

where PIntro corresponds to the incidence (i.e., the proportion of newly infected herds), and PostPInf $f_{t-1}$ is the herd's posterior probability of infection (i.e., 1 PostPFree $\left._{t-1}\right)$. The length of $t$ was set to 3 mo, whereas PIntro was based on results reported by Nielsen and Dohoo (2012) and the prevalence of positive herds in the region. The PIntro was set to 0.003 per time period in a low-prevalence region (mainland Sweden, prevalence $\approx$ 0.01 ), and to 0.02 in a high-prevalence region (Öland, prevalence $\approx 0.2$ ). These calculations were made for 3 different scenarios: (1) HSe based on repeated bulk milk sampling, (2) HSe based on repeated fecal culture of all animals in a herd, and (3) HSe based on repeated sampling with 20 serological samples in each age group and a bulk milk sample.

\section{Surveillance Data}

Surveillance data from recent surveillance activities were compiled and summarized for comparison with simulation results

Comparison of Boot and Dust Swabs to Fecal Samples. To evaluate more convenient herd sampling than the standard sampling within the Salmonella control program, the National Veterinary Institute instructed veterinarians to complement the standard sampling with environmental samples consisting of boot and dust swabs. The standard sampling consisted of individual fecal samples from all cattle in the herd, which were cultured individually or pooled (with a maximum of 5 individuals in the same pool). Composite pat samples were collected when individual samples could not be collected, due to practical or safety reasons. These were taken by collecting feces from several pats equal to the number of animals in the pen, with a maximum of 10 pats represented in each sample. Boot swabs were taken by fitting gauze, moisturized with phosphate buffer or saline solution, on the outside of disposable boot protectors on each boot. Thereafter, the sampler walked with small steps back and forth covering the place where the animals were kept, using 1 set of gauze for a maximum of 50 animals. The gauze was rotated during sampling to achieve complete staining. Dust samples were taken by swiping dusty surfaces of the stable environment, such as gates and walls, with moisturized sterile paper clothes $(23 \times 23 \mathrm{~cm})$, each sample consisting of 3 paper clothes. Complementary sampling, with boot or dust swabs, was performed in 47 herds with 305 groups of animals from 2008 to 2016. These groups were not confined to age categories, but rather to groups of animals with some sort of physical separation. All samples were taken indoors, from animals of all ages, with varied housing and bedding. The culture method used was modified semi-solid Rappaport Vassiliadis (MSRV) selective enrichment medium (Oxoid AB, Thermo Fisher Scientific, Uppsala, Sweden) according to ISO (2002). Groups with at least 1 culture-positive sample were considered to be testpositive. The Salmonella serotypes identified in the sampled herds were Dublin ( $\mathrm{n}=29)$, Typhimurium (n 
$=11)$, Reading $(\mathrm{n}=3)$, monophasic Typhimurium $(\mathrm{n}$ $=2)$, Aarhus $(\mathrm{n}=1)$, and Infantis $(\mathrm{n}=1)$.

Serological Testing of Beef Herds. Swedish Performance Testing of Beef Breeds (Gunnarp, Sweden) is a company that performs yearly evaluation of pedigree bulls. In 2015, this company required Salmonella testing in selling herds before bulls were accepted to the test station. According to the company's standard instruction (Helena Stenberg, Viking Genetics, Gunnarp, Sweden, personal communication), the bull calves and their dams had to be separated from other animals in the herd for at least $2 \mathrm{wk}$ before introduction to the test station, sometimes in company with additional dams and calves. Serum samples were taken from all animals in the separated group, in total 570 samples in 99 different herds, ranging from 1 to 34 sampled animals in each herd. In all herds with at least 1 test-positive sample [i.e., the corrected optical density $(\mathrm{ODC} \%)$ was $\geq 20$ ], follow-up fecal sampling was performed in addition to follow-up serological sampling. In total, 332 followup blood samples were taken in 9 herds. All samples were analyzed with PrioCHECK Salmonella Ab bovine ELISA (including Salmonella Dublin O-antigens 1, 9, 12 and Salmonella Typhimurium O-antigens 1, 4, 5, 12; Thermo Fisher Scientific, Waltham, MA). This test primarily detects antibodies caused by infections with Salmonella Dublin or Typhimurium; however, antibodies caused by infection with other serotypes with common O-antigens are likely to cross-react in this test. Hereafter, this test is referred to as the Bovine ELISA. Fecal culture was performed by the MSRV method.

Bulk Milk Testing in the Safe Trade Program. Since 2011, the Swedish dairy association, Växa Sverige, runs a program called Safe Trade. This program offers subscriptions on bulk milk testing for several diseases every third month. The Bovine ELISA for detection of Salmonella infection is one of the tests used. From 2011 until 2016, a total of 181 herds were sampled within the program, 104 of these were sampled $\geq 4$ times and 44 were sampled throughout the period. Whenever a bulk milk sample was test-positive (i.e., the ODC\% was $\geq 20$ ), follow-up sampling was performed by the Swedish Board of Agriculture (Jönköping, Sweden). In 15 herds with a positive bulk milk sample, follow-up samples were collected within 6 to $90 \mathrm{~d}$ (median $26 \mathrm{~d}$ ). In total, 12 bulk milk samples were taken in 12 of the test-positive herds, and 7 to 20 serum samples from calves 3 to 6 mo were taken in 14 of the test-positive herds. Bulk milk samples and serum samples were tested with the Bovine ELISA and fecal samples were cultured using the MSRV method.

Serological Testing of Calves in the Safe Trade Program. In the Safe Trade program, 4 consecutive negative samples (ODC\% <20) are required for a dairy herd to achieve a Salmonella-free status, equivalent to a time span of 9 mo. The program also offers an opportunity to obtain Salmonella-free status after 1 sampling by complementing the bulk milk sample with serum samples from ten 3 - to 6-mo-old calves. From 2011 until 2016, 886 calves in 86 herds were sampled in this fast track of the program. These samples were analyzed with the Bovine ELISA and samples with an ODC\% $<20$ were considered test-positive. One method to estimate specificity of a test is to sample a low-prevalence population and assume that any positive test result is a false-positive. These test results were used to estimate specificity based on the assumption of disease-free status of these calves.

Statistical Analyses. Ninety-five percent confidence intervals of proportions of positive samples and positive herds were calculated using a binomial distribution. When comparing proportions, Fisher's exact test was used when the expected cell frequency was $<10$ in at least 1 cell, otherwise the chi-squared test was used.

\section{RESULTS}

\section{Test Performance at Group Level}

The GrSe, PPV, and NPV for different group sizes and relevant samples sizes are presented for individual serological testing in Table 2 and for individual bacteriological testing in Table 3. Note that, by definition, GrSe is not dependent on the prior probability of infection. To reach a GrSe of 0.95 in a group of 100 animals, the sample size required for serological testing was 24 . For bacteriological testing, the highest GrSe achieved was 0.83 when sampling all animals. For a group of 50 animals, 22 samples for serology were required to achieve a GrSe of 0.95. For bacteriological testing, the GrSe was 0.66 when all animals in a group with 50 animals were tested.

The PPV was $<0.05$ for all serological sampling based on 5 animals or more in low-prevalence regions. The corresponding estimates for high-prevalence regions were all $<0.52$. For groups with a nonconfirmed suspicion of infection (PriorPInf $=0.5$ ), the $P P V$ was 0.81 for sampling of 5 animals and $\leq 0.71$ for sampling of 20 animals. The PPV increased markedly when 2 positive animals instead of 1 were used as a threshold for considering the group as positive (Table 4). All types of bacteriological sampling had a PPV of 1 , as specificity was assumed to be perfect.

The NPV from testing groups in low-prevalence regions was 0.994 to 1.000 based on all types of sam- 


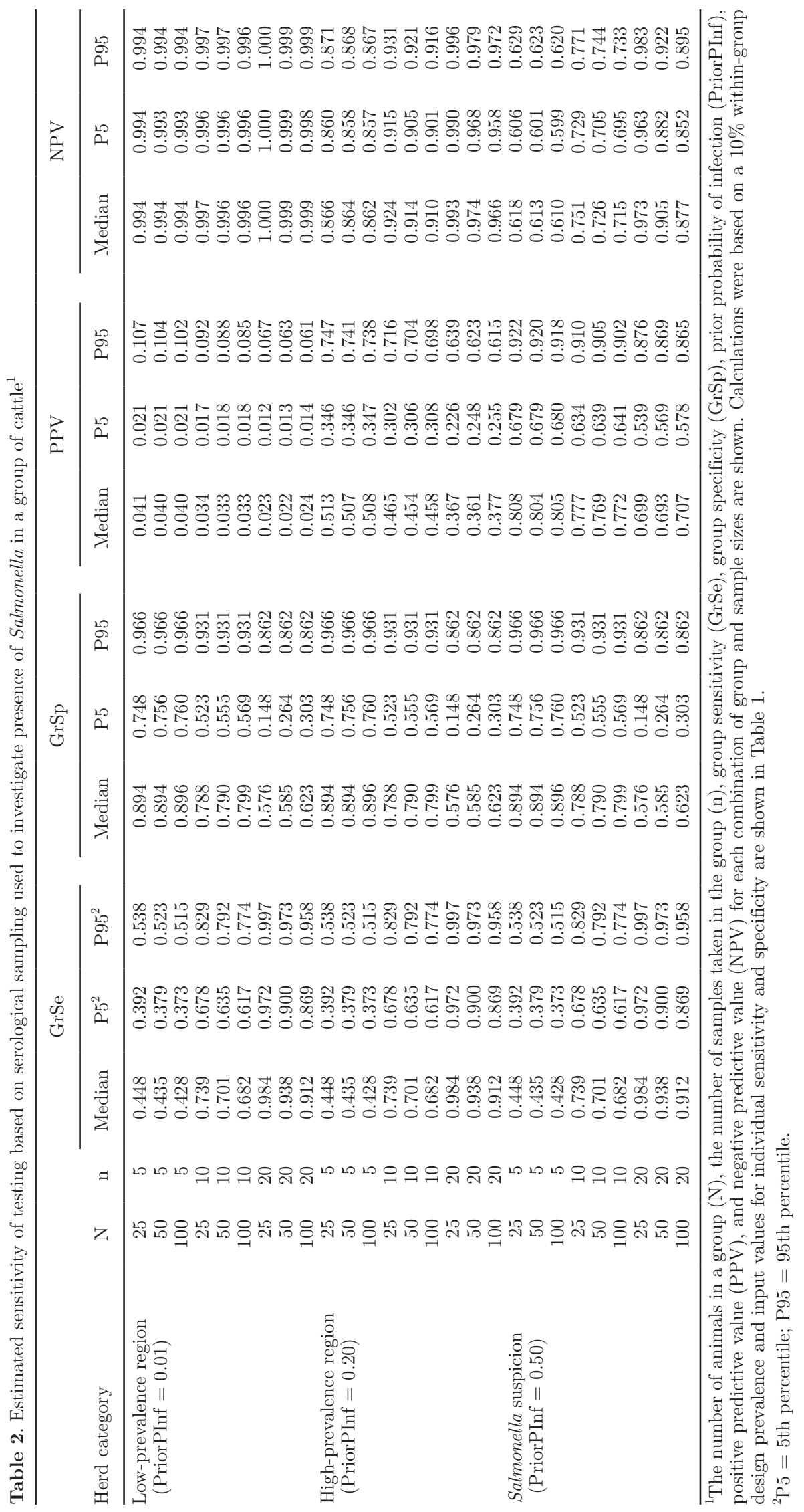


Table 3. Estimated sensitivity of testing based on fecal sampling for culture used to investigate presence of Salmonella in a group of cattle ${ }^{1}$

\begin{tabular}{|c|c|c|c|c|c|c|c|c|}
\hline Herd category & $\mathrm{N}$ & $\mathrm{n}$ & \multicolumn{3}{|c|}{ GrSe } & \multicolumn{3}{|c|}{ NPV } \\
\hline \multirow{6}{*}{$\begin{array}{l}\text { Low-prevalence region } \\
(\text { PriorPInf }=0.01)\end{array}$} & 25 & 10 & 0.226 & 0.135 & 0.310 & 0.992 & 0.991 & 0.993 \\
\hline & 100 & 10 & 0.141 & 0.085 & 0.195 & 0.991 & 0.991 & 0.992 \\
\hline & 25 & 25 & 0.510 & 0.319 & 0.667 & 0.995 & 0.993 & 0.997 \\
\hline & 50 & 25 & 0.386 & 0.240 & 0.510 & 0.994 & 0.992 & 0.995 \\
\hline & 100 & 50 & 0.556 & 0.367 & 0.696 & 0.996 & 0.994 & 0.997 \\
\hline & 100 & 100 & 0.832 & 0.618 & 0.936 & 0.998 & 0.996 & 0.999 \\
\hline \multirow{5}{*}{$\begin{array}{l}\text { High-prevalence region } \\
(\text { PriorPInf }=0.20)\end{array}$} & 25 & 10 & 0.226 & 0.135 & 0.310 & 0.838 & 0.822 & 0.853 \\
\hline & 50 & 10 & 0.169 & 0.101 & 0.233 & 0.828 & 0.817 & 0.839 \\
\hline & 100 & 10 & 0.141 & 0.085 & 0.195 & 0.823 & 0.814 & 0.832 \\
\hline & 25 & 25 & 0.510 & 0.319 & 0.667 & 0.891 & 0.855 & 0.923 \\
\hline & 50 & 25 & 0.386 & 0.240 & 0.510 & 0.867 & 0.840 & 0.891 \\
\hline \multirow{8}{*}{$($ PriorPInf $=0.50)$} & 50 & 10 & 0.169 & 0.101 & 0.233 & 0.546 & 0.527 & 0.566 \\
\hline & 100 & 10 & 0.141 & 0.085 & 0.195 & 0.538 & 0.522 & 0.554 \\
\hline & 25 & 25 & 0.510 & 0.319 & 0.667 & 0.671 & 0.595 & 0.750 \\
\hline & 50 & 25 & 0.386 & 0.240 & 0.510 & 0.620 & 0.568 & 0.671 \\
\hline & 100 & 25 & 0.323 & 0.200 & 0.428 & 0.596 & 0.556 & 0.636 \\
\hline & 50 & 50 & 0.657 & 0.438 & 0.808 & 0.745 & 0.640 & 0.839 \\
\hline & 100 & 50 & 0.556 & 0.367 & 0.696 & 0.693 & 0.613 & 0.767 \\
\hline & 100 & 100 & 0.832 & 0.618 & 0.936 & 0.856 & 0.723 & 0.940 \\
\hline
\end{tabular}

${ }^{1}$ The number of animals in a group $(\mathrm{N})$, the number of samples taken in the group (n), group sensitivity (GrSe), prior probability of infection (PriorPInf), and the negative predictive value (NPV) for each combination of group and sample size are shown. Calculations were based on a $5 \%$ within-group design prevalence and input values for individual sensitivity are shown in Table 1. An animal-level specificity of 1 was used for culture; this is why group specificity and positive predictive value values not are shown, as they consequently will be equal to 1.

${ }^{2} \mathrm{P} 5=5$ th percentile; $\mathrm{P} 95=95$ th percentile.

pling; however, the difference between the PriorPFree and the NPV was only 0.004 to 0.010 . To reach an NPV of 0.95 with serological sampling in groups of 100 animals in high-prevalence regions or in groups with a suspicion of infection, 17 and 29 samples, respectively, were required. For bacteriological sam- pling, the corresponding sample size was 90 samples for a group in a high-prevalence region. With a suspicion of infection, an NPV of 0.95 was not achieved even if all animals were sampled. Using 2 animals as threshold for serology decreased the NPV by 0.002 to 0.142 (Table 4).

Table 4. The median positive and negative predictive value (PPV and NPV) of test results based on serological testing in groups of cattle, using either 1 or 2 test-positive animals as a threshold for considering the group positive $^{1}$

\begin{tabular}{|c|c|c|c|c|c|c|}
\hline \multirow[b]{2}{*}{ PriorPInf } & \multirow[b]{2}{*}{$\mathrm{N}$} & \multirow[b]{2}{*}{$\mathrm{n}$} & \multicolumn{2}{|c|}{ PPV } & \multicolumn{2}{|c|}{ NPV } \\
\hline & & & 1 positive & 2 positive & 1 positive & 2 positive \\
\hline 0.01 & 100 & 5 & 0.040 & 0.256 & 0.994 & 0.991 \\
\hline 0.01 & 100 & 10 & 0.033 & 0.206 & 0.996 & 0.993 \\
\hline 0.01 & 100 & 20 & 0.024 & 0.130 & 0.999 & 0.996 \\
\hline 0.20 & 100 & 5 & 0.508 & 0.895 & 0.862 & 0.813 \\
\hline 0.20 & 100 & 10 & 0.458 & 0.865 & 0.910 & 0.845 \\
\hline 0.20 & 100 & 20 & 0.377 & 0.787 & 0.966 & 0.917 \\
\hline 0.50 & 100 & 5 & 0.805 & 0.972 & 0.610 & 0.521 \\
\hline 0.50 & 100 & 10 & 0.772 & 0.963 & 0.715 & 0.577 \\
\hline 0.50 & 100 & 20 & 0.707 & 0.937 & 0.877 & 0.734 \\
\hline
\end{tabular}

${ }^{1}$ Results are shown for different group size (N), sample size (n), and prior probability of infection (PriorPInf), and input values for individual sensitivity and specificity are shown in Table 1. 


\section{Herd Probability of Freedom}

The HSe and PostPFree for different sampling combinations when used to detect presence of Salmonella in a herd where the infection is present in at least 2 out of 3 age groups are shown in Table 5. Among the combinations investigated, 20 serological samples in each age group plus a bulk milk sample gave the highest estimate of HSe, 0.949 (5th and 95th percentiles = 0.943 and 0.953$)$. This result applies regardless of the value of PriorPFree, as HSe is independent of PriorP-
Free. The combination of fecal sampling of all animals and a bulk milk sample resulted in almost as high HSe, 0.911 (5th and 95th percentiles $=0.835$ and 0.942). For an average herd from a region where prevalence is known to be low, the PriorPFree of 0.99 was already high, and after sampling with high HSe (0.949) the PostPFree was 0.999 (5th and 95th percentiles $=0.999$ and 1.000). For this sampling strategy in herds with a PriorPFree of 0.80 and 0.50 , the PostPFree was 0.987 and 0.951 , respectively. For sampling to detect just 1 out of 3 age groups (Table 6 ), the highest estimate of

Table 5. Herd sensitivity (HSe) and posterior probability of freedom (PostPFree) for cattle herds with different prior probability of freedom (PriorPFree) and different sampling strategies used to detect Salmonella infection in a herd when present in at least 2 out of 3 age groups ${ }^{1}$

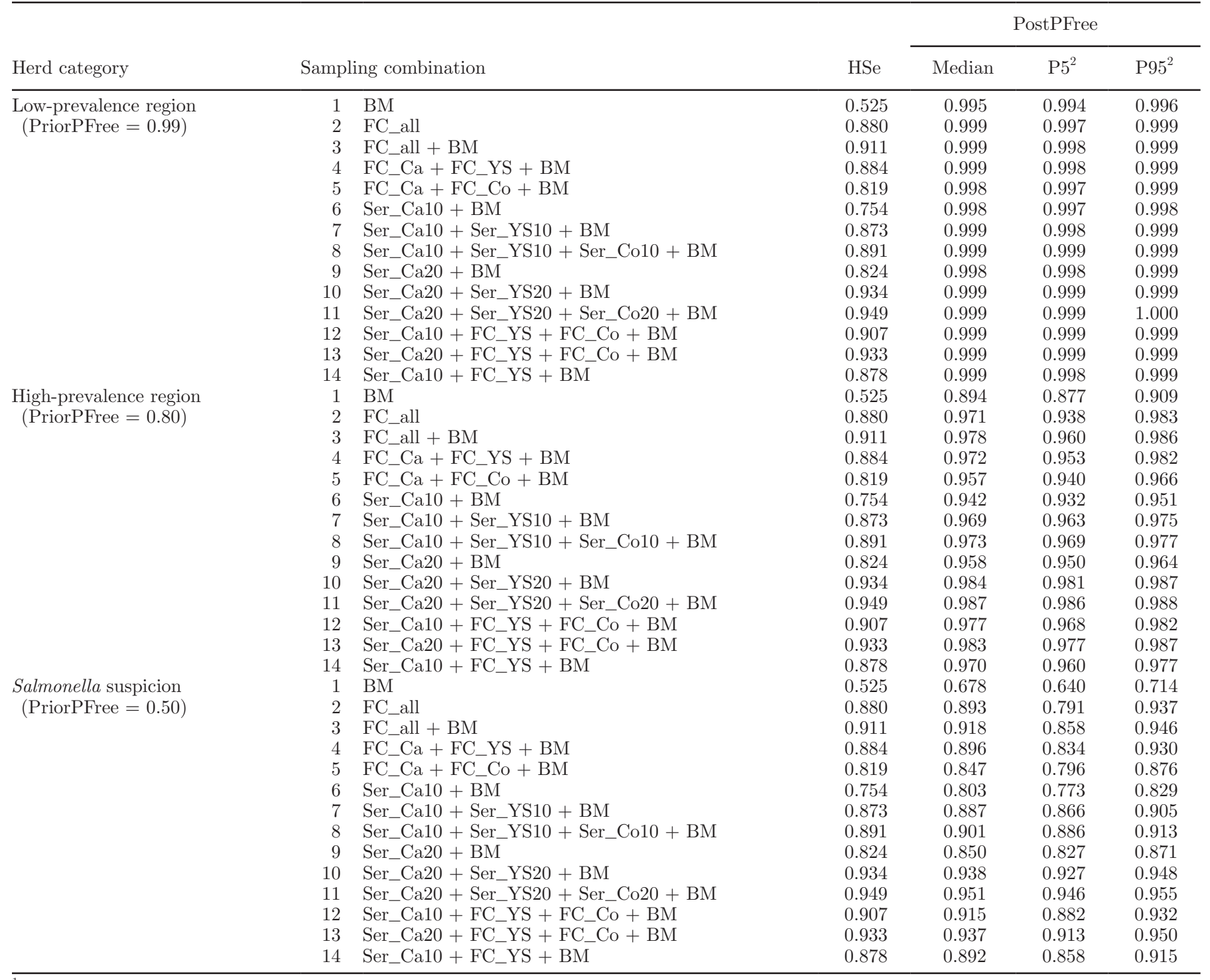

${ }^{1}$ The alternatives included sampling of the 3 age categories [cows (Co), young stock (YS), and calves (Ca)], using sampling of bulk milk (BM), or different sample sizes (all, or number) of fecal sampling for culture (FC) and serological sampling for antibody analyses (Ser).

${ }^{2} \mathrm{P} 5=5$ th percentile; P95 $=95$ th percentile. 
Table 6. Herd sensitivity (HSe) and posterior probability of freedom (PostPFree) for cattle herds with different prior probability of freedom (PriorPFree) and different sampling strategies used to detect Salmonella infection in a herd when present in at least 1 out of 3 age groups ${ }^{1}$

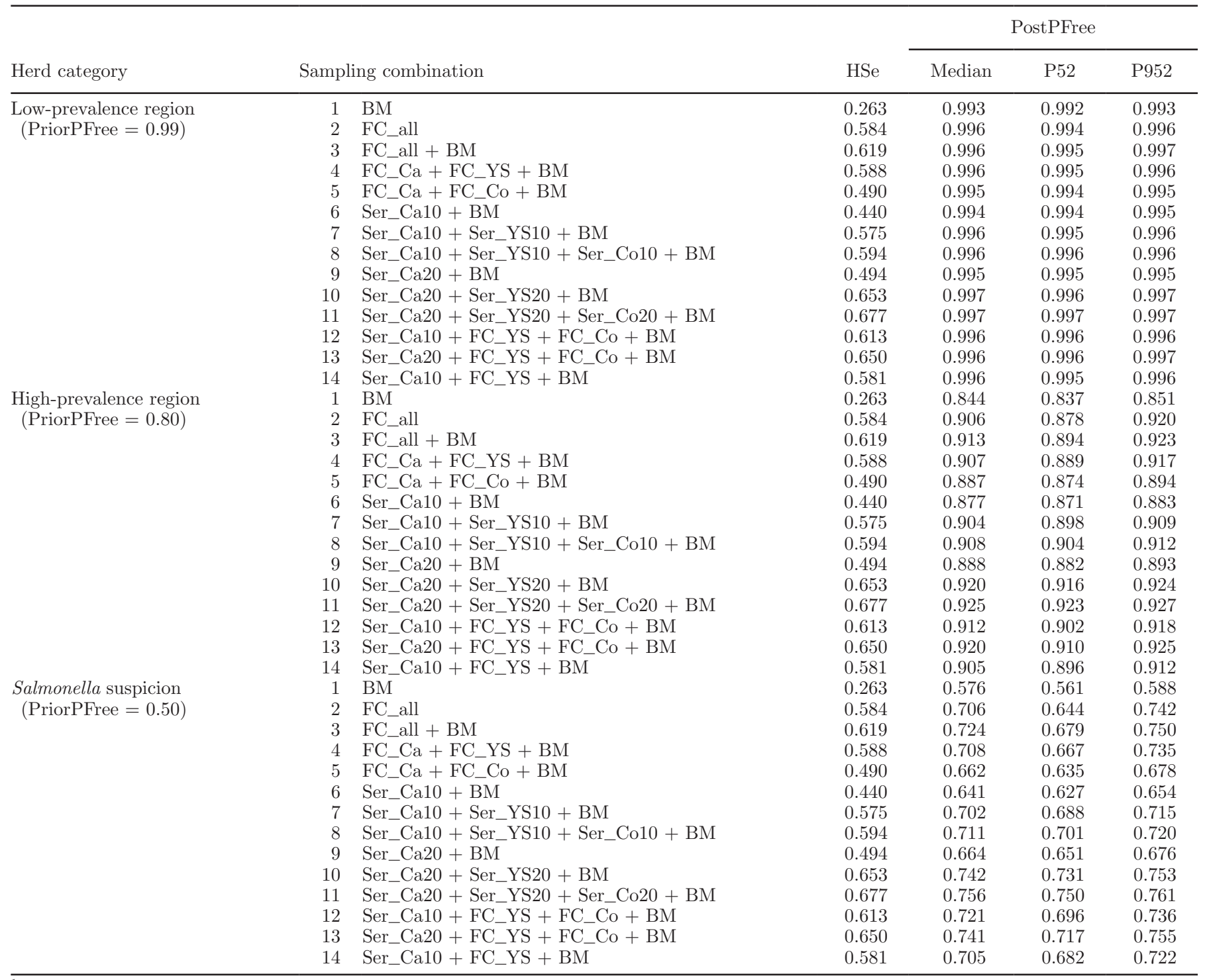

${ }^{1}$ The alternatives included sampling of the 3 age categories [cows (Co), young stock (YS), and calves (Ca)], using sampling of bulk milk (BM), and/or different sample size (all, or number) in fecal sampling for culture (FC) and serological sampling of individual animals (Ser).

${ }^{2} \mathrm{P} 5=5$ th percentile; $\mathrm{P} 95=95$ th percentile

HSe was 0.677, and PostPFree with this HSe was 0.997 for herds in low-prevalence regions (PriorPFree $=0.99$ ) and 0.925 and 0.756 for herds with a PriorPFree of 0.80 and 0.50 , respectively.

A herd's estimated PostPFree from repeated sampling of bulk milk and feces, respectively, given different prevalences (PriorPInf) and incidences (PIntro) is shown in Figure 1. The maximum PostPFree for testing based on repeated fecal sampling of all animals, or based on repeated bulk milk sampling, was within the range of 0.981 to 0.997 , depending on PriorPInf. For herds in high-prevalence regions, it took either 2 rounds of fecal sampling of all animals or 7 rounds of bulk milk sampling to reach this level. For herds with a suspicion of infection, the corresponding numbers were 3 and 9 , respectively. For herds in low-prevalence regions, the maximum PostPFree was $>0.99$ regardless of sampling strategy, and this level of freedom was reached after 1 testing.

\section{Summary of Surveillance Results}

Comparison of Boot and Dust Swabs to Fecal Samples. Both boot swabs and fecal samples were 
A)

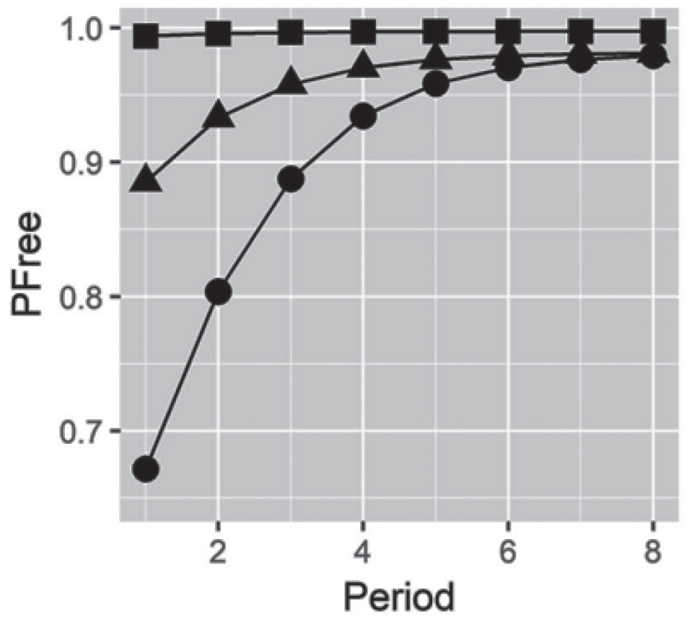

B)

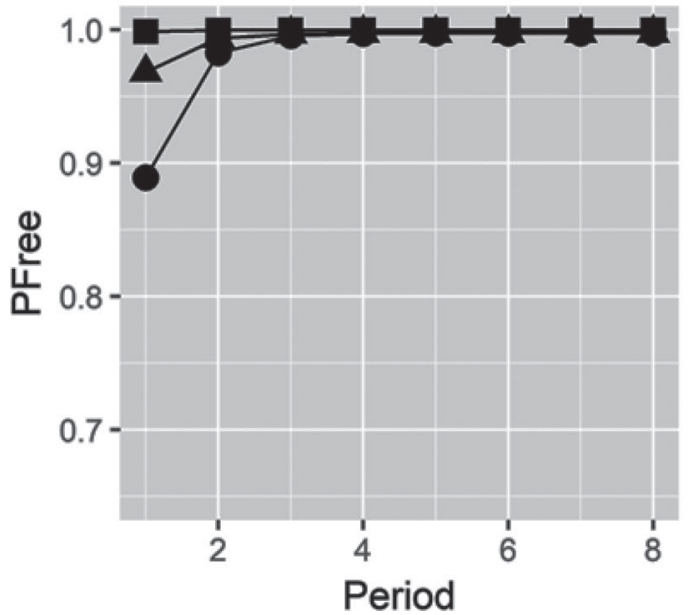

C)

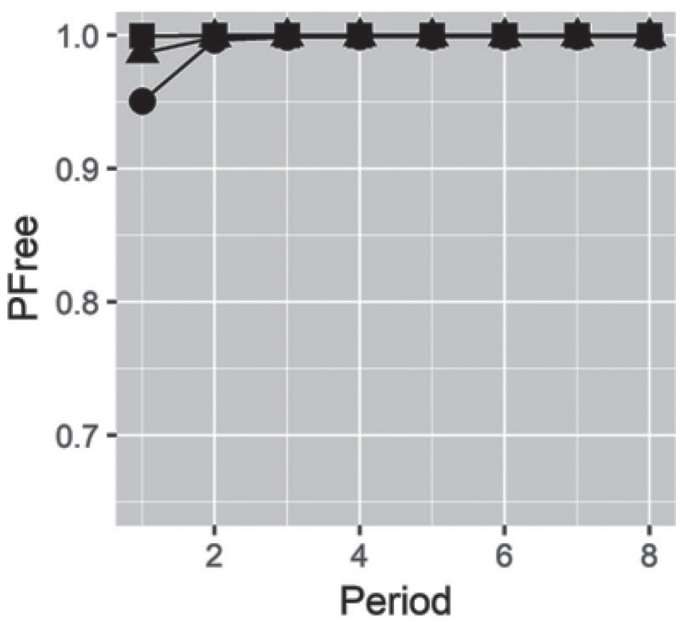

Figure 1. A herd's probability of freedom from Salmonella (PostPFree; y-axis) after repeated testing based on (A) sampling of bulk milk

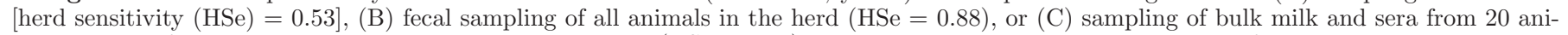

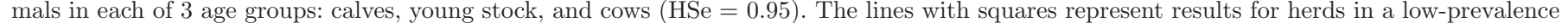

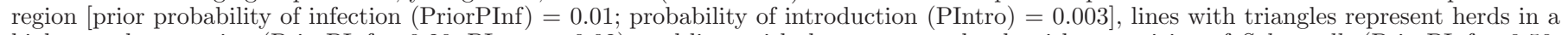
high-prevalence region (PriorPInf $=0.20$, PIntro $=0.02)$, and lines with dots represent herds with a suspicion of Salmonella $($ PriorPInf $=0.50$, PIntro $=0.02)$. Each square, triangle, or dot represents the discounted PostPFree after each new sampling every 3-mo period (x-axis).

collected in 168 groups of animals in 40 herds. Ninetyone groups had positive boot swabs and 90 groups had positive fecal samples (Table 7 ). Of the 90 groups with positive fecal samples, 74 also had positive boot swabs, a proportion of $82 \%$ ( $\mathrm{CI}=73-89 \%)$. The proportion of positive boot swabs was similar for groups with individual fecal samples and groups with composite fecal samples.

Dust and fecal samples were collected in 125 groups in 34 herds (Table 7). Fifty-nine groups had positive dust swabs, and 71 groups had positive fecal samples. Of the 71 positive groups based on fecal sampling, 48 also had positive dust swabs, a proportion of $67 \%$ (CI $=55-78 \%$ ).

All types of samples (i.e., fecal samples and boot and dust swabs) were collected in 117 groups of cattle. Of these, each type of sample had at least 1 culturepositive result in 42 groups, and all samples of all types were negative in 34 groups. In Table 8 , all combinations of results are shown.

Serological Testing of Beef Herds. In the initial sampling, the proportion of positive herds were 9 out of $99(9 \%, \mathrm{CI}=4-17 \%)$, and the number of positive samples were 9 out of $570(1.6 \%, \mathrm{CI}=0.7-3 \%)$. The 
Table 7. Two by two tables showing Salmonella test results at group level from surveillance activities in Sweden 2008-2016

\begin{tabular}{llllllllll}
\hline & & \multicolumn{3}{c}{ Boot } & & \multicolumn{3}{c}{ Dust } \\
\cline { 3 - 5 } \cline { 7 - 9 } Item & Result & neg & pos & total & & neg & pos & total \\
\hline Fecal & neg & 61 & 17 & 78 & & 43 & 11 & 54 \\
& pos & 16 & 74 & 90 & & 23 & 48 & 71 \\
& total & 77 & 91 & 168 & & 66 & 59 & 125 \\
Boot & neg & & & & & 52 & 9 & 61 \\
& pos & & & & & 20 & 56 & 76 \\
& total & & & & & 72 & 65 & 137 \\
\hline
\end{tabular}

${ }^{1}$ The tables compare positive (pos) and negative (neg) test results based on fecal sampling (Fecal) consisting of individual or composite fecal samples, boot swabs (Boot), and dust swabs (Dust). All types of samples were not collected in all groups of cattle.

number of individual serological samples at follow-up varied between 8 and 163 (average $=37$ ). Seven of the 9 herds where follow-up sampling was performed had at least 1 positive sample $(78 \%, \mathrm{CI}=40-97 \%)$, with 1 to 10 positive samples in each herd. The proportion of positive samples at follow-up was 21 out of $332(6 \%$, $\mathrm{CI}=4-32 \%)$. We found a significantly higher proportion of test-positive herds $(P=0.001)$ and test-positive samples $(P<0.001)$ on follow-up compared with the initial sampling. All culture results from fecal sampling were negative at follow-up.

Bulk Milk Testing in the Safe Trade Program. In total, 15 of the 181 herds $(8 \%, \mathrm{CI}=4-13 \%)$ tested positive in at least 1 bulk milk sample. Of these herds, 4 had an OCD $\%>35$. At follow-up, 7 of 12 herds had a positive bulk milk result ( $58 \%, \mathrm{CI}=27-85 \%)$. In herds where follow-up samples of calves were taken, 7 out of 14 herds $(50 \%, \mathrm{CI}=23-77 \%)$ had at least 1 positive calf. In summary, 9 of the 15 positive herds $(60 \%$, CI $=32-84 \%$ ) had a positive bulk milk sample or at least 1 positive calf at follow-up. Only 1 of the 15 herds had a positive fecal culture. The proportion of bulk milk-

Table 8. Test results at group level from groups $(\mathrm{n}=117)$ where all types of samples were collected (i.e., fecal samples consisting of individual or composite fecal samples, boot swabs, and dust swabs); a positive result $(+)$ means that Salmonella was cultured from at least 1 of the samples of that type

\begin{tabular}{lllc}
\hline \multicolumn{2}{l}{ Source of culture } & & \\
\hline Fecal & Boot & Dust & $\begin{array}{c}\text { Number of groups } \\
\text { with result }\end{array}$ \\
\hline+ & + & + & 42 \\
+ & + & - & 12 \\
+ & - & - & 8 \\
+ & - & + & 4 \\
- & + & + & 5 \\
- & + & - & 7 \\
- & - & - & 34 \\
- & - & + & 5 \\
\hline
\end{tabular}

positive herds at follow-up was significantly higher than the proportion of positive herds among all affiliated herds $(P=0.001)$.

Serological Testing of Calves in the Safe Trade Program. Eight of 886 calves $(0.9 \%$, CI $=0.3-1.2 \%)$ sampled within the Safe Trade program tested positive; these were from 8 of 86 herds in total $(9 \%, \mathrm{CI}=4-18 \%)$. Based on these results, assuming that the calves were free from infection, the individual diagnostic specificity of the Bovine ELISA was 0.991 (CI $=0.982-0.996$ ).

\section{DISCUSSION}

We compared the efficiency of different sampling strategies for detection of Salmonella in cattle herds. Our results show that sampling strategies need to be adapted to the risk of infection in different herds and to the purpose of testing.

Regardless of sampling strategy, the added value of testing animals in a low-prevalence region, such as mainland Sweden, may be very low in contrast to the added value of testing in a region where Salmonella is endemic. Based on this, it may be more efficient to focus resources on performing national or regional screenings on a regular basis than performing extensive sampling in individual herds in low-prevalence regions. In this way, it can be demonstrated that the probability of infection remains low. The opposite is true for herds with a higher probability of infection (e.g., herds in regions with a higher prevalence), with a history of confirmed Salmonella infection, or animal trade contacts with infected herds. In these herds, the PPV of test results are considerably higher. Based on our results, sampling these categories of herds will be useful (e.g., in programs for safe trade). To achieve the same level of confidence in freedom from Salmonella as for herds in low-prevalence regions, a sampling strategy with a higher herd sensitivity or repeated sampling is needed.

Our results showed that the use of serological testing for detection of Salmonella in a herd in a low-prevalence region will give very low PPV. In fact, even if the diagnostic specificity on animal level is high (i.e., 0.99 ), there is a high probability that a positive test result is false. In addition, the surveillance data that we compiled showed that some dairy and beef herds have a higher number of positive serum samples than expected. According to Swedish legislation, cattle must have access to pasture in the summer months, and it is likely that some grazing cattle are exposed to Salmonella, as Salmonella can persist in a contaminated environment (Wales and Davies, 2012). However, negative culture results at follow-up in all but 1 of the tested herds, rather low ODC\% values (20-35) in the bulk milk 
tests, and a small proportion of seropositive animals at follow-up suggest that these were low-grade infections that may not require control measures. If consequences of positive test results are severe (e.g., the herd is put under restrictions), both false test positive results as well as detecting herds with low-grade exposure (but no circulating infection) are undesirable. The specificity can be increased by follow-up sampling or by increasing the ELISA cutoff value. The PPV at group level can also be improved by applying a threshold of 2 positive animals instead of 1 . We showed that a threshold of 2 positive animals can be used without substantially decreasing the NPV.

In situations where false-positive test results need to be avoided, testing based on culture from fecal samples may seem appealing, as almost perfect specificity exclude such results. However, sampling strategies based on this testing will often have low sensitivity and the probability that test-negative groups are infected may be high, particularly for Salmonella Dublin infections. This was shown in a Danish study (Nielsen, 2003), where individual fecal samples were collected from all animals in 29 dairy herds and cultured in pools of 5. Samples were collected at 5 occasions 3 mo apart. Salmonella Dublin was isolated, at least once, from 14 of these herds. In all, these 14 herds were sampled at 68 occasions, but culture-positive samples were only found on 40 of these occasions. Despite likely ongoing infection of Salmonella Dublin in these herds, fecal cultures were frequently negative. This highlights the importance of using or supplementing surveillance with serology, particularly when Salmonella Dublin is the serotype of interest. Bulk milk samples also have a limited HSe but are not expensive and are convenient to collect. Our results show that repeated testing can compensate for low HSe in most types of herds with a varying number of sampling occasions. A high probability of freedom could also be achieved with repeated sampling in a high-prevalence region. Our results of repeated sampling are in line with results by Warnick et al. (2006), who estimated that $99 \%$ of the herds classified as probably negative from infection were not infected based on 4 bulk milk samples in a region with a prevalence of $15 \%$.

Our estimates of sensitivity at group level show that a large number of samples are needed for sampling strategies based on individual fecal sampling. However, based on the summary of results from recent surveillance activities, sampling based on boot swabs may be a cost-efficient alternative. We found that boot swabs are almost as efficient as the fecal sampling used within the Swedish control program to identify culture-positive groups of animals. In a previous study, Lombard et al. (2012) evaluated different types of sampling in dairy herds and found that using composite samples had a sensitivity of $85 \%$ compared with individual fecal samples that were cultured individually, whereas pooled individual samples had a sensitivity of $92 \%$ compared with individually cultured samples (i.e., the use of pooled or composite fecal samples will cause reduced sensitivity). In the current study, we compared boot swabs to the samples routinely taken in Swedish cattle herds with a suspicion of Salmonella (i.e., a mixture of pooled individual and composite fecal samples). The results indicated that this standard sampling can be replaced with boot swabs without any substantial loss of sensitivity in identifying Salmonella-infected herds. This agrees with a recent study evaluating sampling strategies for verotoxigenic Escherichia coli in cattle, where boot swabs were found to be an efficient sampling method (Widgren et al., 2013). Boot swabs have also been found to be an efficient sampling method in poultry (Skov et al., 1999).

Our defined purpose for the sampling strategies investigated was to collect information about the presence or absence of circulating Salmonella in a herd. In other words, the true Salmonella status of individual animals was considered of less interest. The proportion of test-positive animals based on bacteriological culture and antibody testing can be expected to differ, as the period of bacterial shedding is considerably shorter than the persistence of antibodies in the blood. The design prevalences chosen for our calculations represent the targets of the assessed sampling strategies. Based on reports in the literature, we estimated that circulating Salmonella infection in a group in most cases would lead to a proportion of at least $5 \%$ of the animals excreting Salmonella, whereas at least $10 \%$ of the animals are likely to have developed an antibody response to Salmonella. Therefore, the design prevalences for fecal sampling and serological sampling were set to these values. For Salmonella serotypes with no O-antigen in common with those included in the tests (Dublin and Typhimurium), the degree of antibody response is unknown, and animals that have been infected with such serotypes cannot be expected to be test-positive on serology. However, Salmonella Dublin and Typhimurium are the dominating serotypes in Europe, and Salmonella Typhimurium is one of the dominating serotypes in North America (Blau et. al., 2005; European Food Safety Authority, 2016). In addition, a large proportion of other serotypes detected in Sweden have O-antigens in common with Salmonella Dublin or Typhimurium and may therefore cross-react in the test (Lewerin et al., 2011).

In our study, the design prevalence at group level was set to 2 out of 3 groups. The contacts between age groups within a herd followed the circular flow of calves 
to young stock, young stock to adult animals, and finally from adult animals back to calves. This means that, over time, Salmonella often spreads to more than one age group, even in herds with good internal biosecurity. A comparison of our simulated results and others' estimates of sensitivity of bulk milk testing (Veling et al., 2002) suggests that the true group prevalence may be, on average, 2 groups out of 3 . Thus, this level of infection is the most probable target for animal health organizations or governmental authorities that want to control or perform surveillance of Salmonella on a population level. However, it should be noted that for newly infected herds infection may be present in just 1 age group, and, as an alternative, a design prevalence of 1 out of 3 was also used in our calculations. Estimates based on this level could be considered in the planning of sampling strategies aiming for a very high confidence in Salmonella freedom in test-negative herds; for example, farmers that need to buy animals but want to minimize the probability of introducing Salmonella in their herd. They could also limit their purchases to animals from herds with a relatively long history of repeated test results or to animals from confirmed lowprevalence regions.

In conclusion, our study highlights the importance of considering a herd's risk of Salmonella upon decisions on sampling. The results can be used when deciding on sampling strategies for different purposes, for example, surveillance or prepurchase testing in cattle herds.

\section{ACKNOWLEDGMENTS}

We thank Catrin Vesterlund-Carlson (National Veterinary Institute, Sweden) for help with compiling results from surveillance activities and Maarten Weber (GD Animal Health, Deventer, the Netherlands) for comments on the manuscript. The study was financially supported by the Swedish Farmers' Foundation for Agricultural Research (Stockholm, Sweden).

\section{REFERENCES}

Ågren, E. C. C., S. Sternberg Lewerin, H. Wahlström, U. Emanuelson, and J. Frössling. 2016. Low prevalence of Salmonella in Swedish dairy herds highlight differences between serotypes. Prev. Vet. Med. 125:38-45.

Blau, D. M., B. J. McCluskey, S. R. Ladely, D. A. Dargatz, P. FedorkaCray, K. E. Ferris, and M. L. Headrick. 2005. Salmonella in dairy operations in the United States: Prevalence and antimicrobial drug susceptibility. J. Food Prot. 68:696-702.

Cameron, A. R., and F. C. Baldock. 1998. Two-stage sampling in surveys to substantiate freedom from disease. Prev. Vet. Med. $34: 19-30$

Carrique-Mas, J. J., J. A. Willmington, C. Papadopoulou, E. N. Watson, and R. H. Davies. 2010. Salmonella infection in cattle in Great Britain, 2003 to 2008. Vet. Rec. 167:560-565.
Dohoo, I., W. Martin, and H. Stryhn. 2009. Screening and diagnostic tests, page 104, Veterinary Epidemiologic Research, 2nd ed. VER Inc., Charlottetown, Prince Edward Island, Canada.

European Food Safety Authority. 2016. The European Union summary report on trends and sources of zoonoses, zoonotic agents and food-borne outbreaks in 2015. Accessed May 29, 2018. http:// onlinelibrary.wiley.com/doi/10.2903/j.efsa.2016.4634/full.

Fossler, C. P., S. J. Wells, J. B. Kaneene, P. L. Ruegg, L. D. Warnick, J. B. Bender, L. E. Eberly, S. M. Godden, and L. W. Halbert. 2005a. Herd-level factors associated with isolation of Salmonella in a multi-state study of conventional and organic dairy farms-I. Salmonella shedding in cows. Prev. Vet. Med. 70:257-277.

Fossler, C. P., S. J. Wells, J. B. Kaneene, P. L. Ruegg, L. D. Warnick, J. B. Bender, L. E. Eberly, S. M. Godden, and L. W. Halbert. 2005b. Herd-level factors associated with isolation of Salmonella in a multi-state study of conventional and organic dairy farms-II. Salmonella shedding in calves. Prev. Vet. Med. 70:279-291.

Hugas, M., and P. A. Beloeil. 2014. Controlling Salmonella along the food chain in the European Union-Progress over the last ten years. Euro Surveill. 19:2-5.

ISO. 2002. ISO6579:2002 E standard: Microbiology of food and animal feeding stuffs - Horizontal method for the detection of Salmonella spp. International Organization for Standardization (ISO), Geneva, Switzerland.

Lewerin, S. S., L. Skog, J. Frössling, and H. Wahlström. 2011. Geographical distribution of salmonella infected pig, cattle and sheep herds in Sweden 1993-2010. Acta Vet. Scand. 53. https://doi.org/ 10.1186/1751-0147-53-51.

Lombard, J. E., A. L. Beam, E. M. Nifong, C. P. Fossler, C. A. Kopral, D. A. Dargatz, B. A. Wagner, M. M. Erdman, and P. J. FedorkaCray. 2012. Comparison of individual, pooled, and composite fecal sampling methods for detection of Salmonella on U.S. dairy operations. J. Food Prot. 75:1562-1571.

Martin, P. A. J., A. R. Cameron, K. Barfod, E. S. G. Sergeant, and M. Greiner. 2007b. Demonstrating freedom from disease using multiple complex data sources 2: Case study - Classical swine fever in Denmark. Prev. Vet. Med. 79:98-115.

Martin, P. A. J., A. R. Cameron, and M. Greiner. 2007a. Demonstrating freedom from disease using multiple complex data sources 1: A new methodology based on scenario trees. Prev. Vet. Med. 79:71-97.

National Veterinary Institute. 2017. Surveillance of infectious diseases in animals and humans in Sweden 2016. Accessed May 29, 2018. http://www.sva.se/smittlage/zoonosrapporter.

Nielsen, L. R. 2003. Salmonella Dublin in dairy cattle - Use of diagnostic tests for investigation of risk factors and infection dynamics. PhD thesis. Department of Large Animal Sciences, The Royal Veterinary and Agricultural University, Copenhagen, Denmark.

Nielsen, L. R. 2012. Salmonella Dublin in cattle. Epidemiology, design and evaluation of surveillance and eradication programmes. DVM thesis. Department of Large Animal Sciences, University of Copenhagen, Denmark

Nielsen, L. R. 2013. Within-herd prevalence of Salmonella Dublin in endemically infected dairy herds. Epidemiol. Infect. 141:2074-2082.

Nielsen, L. R., and I. Dohoo. 2012. Survival analysis of factors affecting incidence risk of Salmonella Dublin in Danish dairy herds during a 7-year surveillance period. Prev. Vet. Med. 107:160-169.

Nielsen, L. R., and A. K. Ersbøll. 2004. Age-stratified validation of an indirect Salmonella Dublin serum enzyme-linked immunosorbent assay for individual diagnosis in cattle. J. Vet. Diagn. Invest. $16: 212-218$.

Nielsen, L. R., N. Toft, and A. K. Ersbøll. 2004. Evaluation of an indirect serum ELISA and a bacteriological faecal culture test for diagnosis of Salmonella serotype Dublin in cattle using latent class models. J. Appl. Microbiol. 96:311-319.

Nielsen, T. D., A. B. Kudahl, S. Ostergaard, and L. R. Nielsen. 2013. Gross margin losses due to Salmonella Dublin infection in Danish dairy cattle herds estimated by simulation modelling. Prev. Vet. Med. 111:51-62. 
Nielsen, T. D., L. R. Nielsen, and N. Toft. 2011. Bayesian estimation of true between-herd and within-herd prevalence of Salmonella in Danish veal calves. Prev. Vet. Med. 100:155-162.

Pires, S. M., A. R. Vieira, T. Hald, and D. Cole. 2014. Source Attribution of Human Salmonellosis: An Overview of Methods and Estimates. Foodborne Pathog. Dis. 11:667-676.

R Development Core Team. 2018. The R Project for Statistical Computing. Accessed Mar. 8, 2018. http://www.R-project.org.

Richardson, A. 1975. Salmonellosis in cattle. Vet. Rec. 96:329-331.

Sergeant, E. 2016. RSurveillance: Design and Analysis of Disease Surveillance Activities. Accessed May 29, 2018. https://CRAN.R -project.org $/$ package $=$ RSurveillance.

Skov, M. N., B. Carstensen, and M. Madsen. 1999. Evaluation of sampling methods for the detection of Salmonella in broiler flocks. J. Appl. Microbiol. 86:695-700.

Swedish Board of Agriculture. 2004. Regulations on control of salmonella in animals (In Swedish. Statens jordbruksverks föreskrifter om bekämpande av salmonella hos djur) SJVFS 2004:2 Saknr K 102, Jönköping, Sweden.

Swedish Board of Agriculture. 2012. Structure of the Swedish cattle industry (In Swedish. Nötkreaturssektorns uppbyggnad). Accessed May 29, 2018. http://www.jordbruksverket.se/omjordbruksverket/ statistik/statistikomr/arkiveradestatistikrapporter. 4 .67e843d911ff9f551db80008895.html.

Veling, J., H. W. Barkema, J. van der Schans, F. van Zijderveld, and J. Verhoeff. 2002. Herd-level diagnosis for Salmonella enterica ssp. enterica serovar Dublin infection in bovine dairy herds. Prev. Vet. Med. 53:31-42.

Wales, A., and R. H. Davies. 2012. Environmental aspects of Salmonella. Pages 399-425 in Salmonella in Domestic Animals. 2nd ed. Barrow, P. and Methner, U. CAB International, Wallingford, United Kingdom.

Warnick, L. D., L. R. Nielsen, J. Nielsen, and M. Greiner. 2006. Simulation model estimates of test accuracy and predictive values for the Danish Salmonella surveillance program in dairy herds. Prev. Vet. Med. 77:284-303.

Wedderkopp, A., U. Strøger, V. Bitsch, and P. Lind. 2001. Testing of bulk tank milk for Salmonella Dublin infection in Danish dairy herds. Can. J. Vet. Res. 65:15-21.

Widgren, S., E. Eriksson, A. Aspan, U. Emanuelson, S. Alenius, and A. Lindberg. 2013. Environmental sampling for evaluating verotoxigenic Escherichia coli O157:H7 status in dairy cattle herds. J. Vet. Diagn. Invest. 25:189-198. 